\title{
Visual Security Protocol Modeling
}

\author{
J. McDermott \\ Center for High Assurance Computer Systems \\ Naval Research Laboratory \\ Washington, DC 20375, USA \\ John.McDermott@NRL.Navy.mil
}

\begin{abstract}
This paper argues that the existing model-driven architecture paradigm does not adequately cover the visual modeling of security protocols: sequences of interactions between principals. A security protocol modeling formalism should be not only well-defined but also support event-based, compositional, comprehensive, laconic, lucid, sound, and complete modeling. Candidate visual approaches from both the OMG's MDA and other more well-defined formalisms fail to satisfy one or more of these criteria. By means of two example security protocol models, we present the GSPML visual formalism as a solution.
\end{abstract}

\section{Categories and Subject Descriptors}

D.2.1 [Requirements/Specifications]: Languages; D.3.3 [Programming Languages]: Concurrent programming structures; D.4.6 [Security and Protection]: Cryptographic controls, Information flow controls

\section{General Terms}

Security, Design, Languages

\section{Keywords}

security protocol, security principal, GSPML

\section{INTRODUCTION}

The model-driven-approach [38] to software construction promises to improve software quality and reduce costs through automatic construction of software from (visual) models. Visual modeling is slowly becoming a common practice for software developers, so the hope is that practitioners will be comfortable with using visual models to design their software. (In this paper, we use the unqualified term modeling to mean visual modeling.)

The force of common practice is defining the model-drivenapproach in terms of the Object Management Group's Model

NSPW 2005 Lake Arrowhead CA USA

(c) 2006 ACM 1-59593-317-4/06/02...\$5.00

Permission to make digital or hard copies of all or part of this work for personal or classroom use is granted without fee provided that copies are not made or distributed for profit or commercial advantage and that copies bear this notice and the full citation on the first page. To copy otherwise, to republish, to post on servers or to redistribute to lists, requires prior specific permission and/or a fee.
Driven Architecture or MDA. The core of the MDA is UML 2.0 [32]. Neither UML 2.0 (henceforth UML) or MDA treats security as much more than a service; there are no models for security per se.

This raises the question of what security-specific aspects of software development, if any, need coverage in this paradigm. This paper argues that there are security-specific issues that cannot be modeled well with existing features of MDA. These issues need adequate coverage in model-driven approaches.

One of the most significant security-specific aspects of software development not covered by the MDA is the security protocol. Security protocols are sequences of allowable interactions between principals. A principal is an entity that participates in a security system. Security protocols are not necessarily about cryptography; one of our examples will model a security protocol that involves no cryptography at all.

The UML candidates for visual modeling of security protocols all have shortcomings. Existing alternatives outside of UML also have similar problems, for various reasons. Some of these difficulties are visual modeling issues and others are semantic issues. One of the most critical semantic requirements for modeling security protocols is the ability to define all traces of a protocol with a single model as opposed to being able to describe any trace with a single model. Explicit definition of all traces is necessary because each bad trace has the potential to become a security flaw. A highly desirable visual modeling feature is event-based modeling, as opposed to state-based modeling. The distinction is that state-based modeling is best for designing reactive behavior while event-based modeling is best for designing interactive behavior. State-based modeling requires us to work with internal computational aspects, such as states or triggers, to construct the traces of a protocol. An event-based modeling paradigm lets us work directly with the external events and traces of a protocol.

\section{MOTIVATION}

The core purpose of visual modeling, as opposed to other forms of modeling, is presentation and understanding. Formal verification, machine-generated implementation, and other automatic processing are probably done better with textbased models. In fact, a good well-defined visual language will always be a form of syntactic sugar [24] for some textbased languge, since the text-based semantics will be needed for execution. So our interest is in security protocol modeling that has good visual properties for presentation and human understanding, without sacrificing soundness that 
supports translation into text-based models. This leads to the following criteria for security protocol modeling:

- The visual formalism should have a well-defined syntax and semantics.

- The visual formalism should be event-based. It should focus on interaction patterns between principals and abstract away from details of internal computations. The importance of this is underscored by the fact that existing security protocol modeling tools use eventbased visual models, rather than state-based models.

- The visual formalism should support models that are compositional. Compositional models are constructed from sub-models that identifiably correspond to the principals of the protocol.

- The visual formalism should suport models that are comprehensive. It should be capable of defining all traces of a protocol by means of a single diagram.

- The visual formalism should suport models that are laconic [15]. A non-laconic model is one where some object or relation in the represented abstraction is modeled more than once. Following Guizzardi [14], a model $\mathcal{M}$ is laconic w.r.t. an abstraction $\mathcal{A}$ if the interpretation mapping from $\mathcal{M}$ to $\mathcal{A}$ is injective.

- The visual formalism should suport models that are lucid [15]. A non-lucid model is one where some object or relation in the model represents more than one object or relation from the modeled abstraction. Following Guizzardi [14], a model $\mathcal{M}$ is laconic w.r.t. an abstraction $\mathcal{A}$ if the representation mapping from $\mathcal{A}$ to $\mathcal{M}$ is injective.

- The visual formalism should suport models that are sound [15]. An unsound model is one where some model object or relation has no corresponding object or relation in the represented abstraction. Following Guizzardi [14], a model $\mathcal{M}$ is sound w.r.t. an abstraction $\mathcal{A}$ if the representation mapping from $\mathcal{A}$ to $\mathcal{M}$ is surjective.

- The visual formalism should suport models that are complete [15]. An incomplete model is one where some object or relation in the represented abstraction has no corresponding model object or relation. Following Guizzardi [14], a model $\mathcal{M}$ is complete w.r.t. an abstraction $\mathcal{A}$ if the interpretation mapping from $\mathcal{M}$ to $\mathcal{A}$ is surjective.

The UML candidates for visual modeling are either not well-defined or they fail to suport comprehensive or laconic models. Visual modeling candidates outside UML are welldefined but are either state-based or fail to suport models that are laconic or comprehensive. The visual interfaces to current security protocol modeling tools also do not provide a formalism that satisifies all of our criteria. These candidates are not necessarily bad but are not suited to visual security protocol modeling, according to one or more of the criteria above. We make these statements without explanation here but present a detailed justification in Section 4.

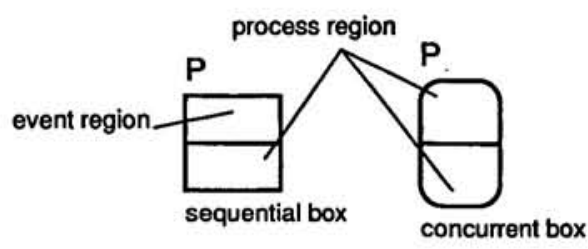

Figure 1: Basic Boxes of GSPML

\section{GSPML}

We present GSPML as a visual security protocol modeling language that satisfies all of the above criteria. (At the time this paper was written, GSPML did not stand as an acronym of any particular name.) The goal of the GSPML alternative is to provide a visual modeling language suitable for the security-specific problem of protocol modeling. The emphasis is on a solid visual model with complete syntax and semantics, rather than tool application via the specific semantics. Given a well-defined visual modeling language, a variety of formal techniques could be used, including many with semantics that differ from the semantics of GSPML (e.g. NPATRL, CAPSL, strand spaces $[12,39]$, or a general LTS).

The GSPML alternative is well-defined, event-based, compositional, comprehensive, and laconic. This is demonstrated by the diagram at the end of this paper (see Figure 11) that defines a complete model of the Yahahlom cryptographic security protocol [5]. This diagram fits on a single page but defines all possible traces of the protocol interacting with a Dolev-Yao intruder. So a single GSPML diagram can define not only all the correct behavior of a protocol but also its behavior under many attacks.

Our presentation here in Section 3 does not define a semantics for the language but provides an introduction and demonstrates the applicability of GSPML. The meaning of well-formed GSPML diagrams is compatible with several forms of process algebra but it is not necessary to understand process algebra in order to understand the GSPML presented in this paper. It is necessary to understand that GSPML models are arrangements of nested rectangles or boxes that define trace generating processes.

In GSPML, every trace-generating process is defined by either a process box or a process box name. (For the rest of this paper we will use the term process and box interchangeably.) There are two major distinctions between boxes: sequential boxes and concurrent boxes, as in Figure 1. A sequential box has rectangular corners and models sequential processes. A concurrent box has round corners and models concurrent processes. A process box name (or more simply, box name) may only appear as a label for a box, or inside a process region of a box. A process region may have only one box or box name in it. Sequential boxes also have event regions that contain the events of a GSPML model. When all of the events in the event region of a sequential box have occurred then the sequential box is replaced by or becomes the box contained or named in the process region below the event region. So GSPML diagrams are read from top to bottom and outside to in.

We present the details of GSPML by examples of its use. We give two examples: first a cryptographic security protocol and then a non-cryptographic security protocol. 


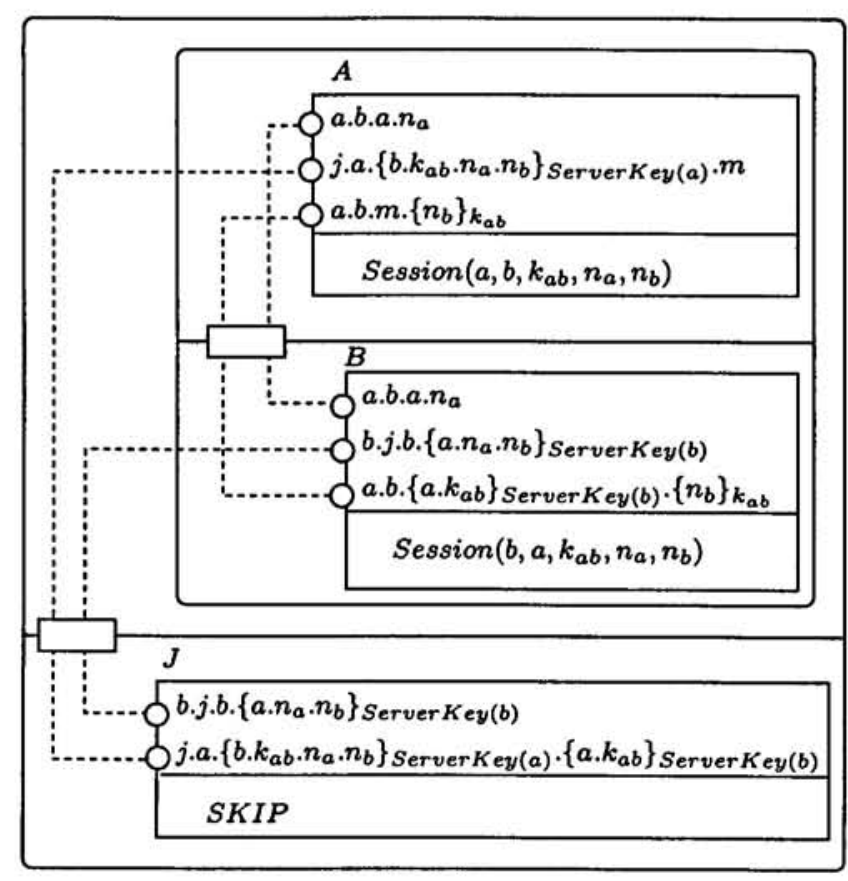

Figure 2: A GSPML Model of One Run of the Yahalom Protocol

\subsection{The Yahalom Protocol}

Our example of a cryptographic security protocol is the Yahalom protocol. The GSPML model is based on the CSP process algebra model presented by Ryan, Schneider, et al. [35]. Readers interested in process algebra modeling of security protocols, as opposed to exposition of the GSPML language, should consult their work.

The Yahalom protocol is used to establish a session key $k_{a b}$ between two principals $A$ and $B$, via a server $J$. Figure 2 shows a simple GSPML diagram of a single run of the protocol, where principal $A$ initiates a session with principal $B$. The protocol run is simplified in the sense that the principals are assumed to be somehow prepared to synchronize on each other's nonces and the session key, in advance of the protocol run. That is, each event contains precisely the nonces $n_{a}, n_{b}$ and session key $k_{a b}$ to make this run work. None of the three named boxes in Figure 2 is defined as being prepared to deal with any possible well-formed nonce or session key.

The goal of Figure 2 is to introduce the protocol, not model it. That is, the GSPML of Figure 2 is playing the role of the usual message sequence diagram used to introduce a cryptographic protocol. So Figure 2 shows that GSPML can be used for explanation as well as definition of security protocols.

Figure 2 provides a good non-trivial first example of GSPML. The two outermost (unnamed, round-cornered) boxes are concurrent boxes that model the concurrent interaction of the principals $A$ and $B$ and the server. Each concurrent box has two process regions. The outermost concurrent box has the next inner concurrent box in one of its process regions and a sequential box named $J$ in its other process region. The sequential box $J$ contains the events that model the protocol steps of the server in a single run of the Yahalom protocol. The second concurrent box contains the sequential boxes named $A$ and $B$ defining the corresponding protocol steps for the initiator and responder.

Each event in a sequential box is denoted by a small circle, called an event symbol, on the left boundary of the box's event region. The top-to-bottom order of the event symbols defines the sequential order of the events for that box. So the events of sequential box $A$ at the top of Figure 2 are

$$
\begin{aligned}
& \text { a.b.a.n } n_{a} \\
& \text { j.a. }\left\{b \cdot k_{a b} \cdot n_{a} \cdot n_{b}\right\}_{\text {ServerKey }(b) \cdot m} \cdot m \\
& \text { a.b.m. }\left\{n_{b}\right\}_{k} a b
\end{aligned}
$$

Sequential boxes communicate or share their events via interface port symbols on enclosing concurrent boxes. Concurrent boxes with interface port symbols are parallel boxes that define communication between their components. An interface port symbol is a small rectangle placed on the boundary between the process regions of a parallel box.

Shared events are connect by synchronization lines. The synchronization lines shown in Figure 2 are an example of concrete synchronization lines because they connect event symbols directly. (There are abstract synchronization lines that do not connect event symbols. They will be presented shortly.)

Concrete synchronization lines depict sharing of the specific events they connect. The events connected by the synchronization lines happen at the same time; they appear as a single event to an outside observer. Synchronization lines may be drawn anywhere that provides clarity while connecting the events, but must pass through the interface port symbol that defines the parallel combination.

In Figure 2 the shared events model the transmission and receipt of a message in the security protocol.

Events in GSPML may have compound names. The event itself is atomic but various information about the event can be represented using a "dot" separator, as in $x . y$ between the name components $x$ and $y$.

In Figure 2's model of a run of the Yahalom protocol, the events have compound names with the first component indicating the sender for that event and the second component indicating the receiver of the event. For example, the first event of box $A$ is the compound event a.b.a. $n_{a}$ : a transmission from source $a$ to destination $b$ of the message $a . n_{a}$. The concrete synchronization line from event a.b.a. $n_{a}$ in box $A$ to the same event in box $B$ models the transmission of message $a . n_{\mathrm{a}}$ by principal $A$ and receipt by principal $B$.

Figure 2 also contains concrete synchronization lines connecting events with different names at source and destination. For example the second event of box $A$ is named $j . a\left\{b . k_{a b} \cdot n_{a} \cdot n_{b}\right\}_{\text {Server Key (a) }} . m$ while the source event in box $J$ is named $j . a\left\{b . k_{a b} . n_{a} . n_{b}\right\}_{\text {ServerKey }(a)} \cdot\left\{a . k_{a b}\right\}_{\text {ServerKey(b) }}$. This aliasing indicates that the source and destination boxes have different interpretations of the shared event. In this case, the initiator (modeled by box $A$ ) cannot read the last component $\left\{a . k_{a b}\right\}_{\text {Server } K e y_{(b)}}$ because it does not have ServerKey(b) so it interprets that component as simply as a sequence of bits $m$.

We can tell which event happens first in the diagram of Figure 2 by noticing that second event of the $B$ box happens at the same time as the first event of the $J$ box, so box $J$ 's first event cannot start the protocol. As the diagram shows it, the first event in the protocol must be the shared first event of boxes $A$ and $B$ : transmission of the message $a . n_{a}$ 
from $a$ to $b$. (It is not always necessary that a unique event in a GSPML diagram be the first event; the first event can be one of several possibilities.)

The Yahalom protocol works as follows: principal $A$ wishes to establish a session with principal $B$ and initiates a run of the protocol by sending its identity $a$ and a nonce $n_{a}$ to principal $B$. This is shown by the synchronization of the first event a.b. $n_{a}$ communicated from the $A$ box to the $B$ box in Figure 2. Box $B$ then sends $a, n_{a}$ and its own nonce $n_{b}$, encrypted under the key ServerKey $(b)$ to the server. This is show in Figure 2 by the synchronization of the second event of the $B$ box with the first event of the $J$ box. The third step of the protocol has the server (box $J$ ) send principal $B$ 's identity $b$, both nonces $n_{a}, n_{b}$, a session key $k_{a b}$, and a message $\left\{a . k_{a b}\right\}_{\text {Server Key }(b)}$ to principal $A$. This is shown in Figure 2 by the synchronization of the second event of the $J$ box with the second event of the $A$ box, where the initiator sees the message $\left\{a . k_{a b}\right\}_{\text {Server Key }(b)}$ simply as a bit string $m$.

In Figure 2 the end of the protocol run is shown by the $J$ box becoming the (constant) process box $S K I P$ that denotes successful termination, while the $A$ and $B$ boxes become parameterized Session boxes that denote the start of a session between principals $A$ and $B$.

\subsection{A Complete GSPML Model}

Defining a complete model of the Yahalom protocol will explain more of the GSPML language and demonstrate that it is event-based, comprehensive, concise, well-defined, and composable. Our complete model follows the Dolev-Yao structure where the intruder acts as the network connecting the principals. The complete GSPML model is shown as Figure 11 at the end of the paper, but we do not use that figure to explain GSPML. Instead, the model is presented beginning from a top-level view. Then components of the complete model are explained, proceeding from simpler constructions to more complex. This will demonstrate the abstraction capabilities of GSPML. The form of our explanation is to introduce different language elements by example. The meaning of each language element is explained first and then the protocol modeling structure is explained based on the meaning.

\subsection{High-Level Model Structure}

Figure 3 shows a top-level view of the model of Figure 11 , with principals $A$ and $B$ as abstract concurrent boxes User(a) and User(b), the server as the abstract concurrent box $\operatorname{Server}(j)$, and the intruder as abstract sequential box Intruder $(X)$. Abstract boxes have no internal regions for events or processes. This use of abstract concurrent and sequential boxes shows the high-level structure of the model without the internal details.

The basic structure of Figure 3 is a parallel box synchronizing the sequential Intruder $(X)$ box with the nested interleaving boxes that model $U \operatorname{ser}(a), U \operatorname{ser}(b)$ and $\operatorname{Server}(j)$. The boxes modeling Intruder $(X), U \operatorname{ser}(a), U \operatorname{ser}(b)$ and $\operatorname{Server}(j)$ are each enclosed by a box drawn with dashed lines, just like a synchronization line. These dashed boxes indicate the use of renaming to map the names of events into other event names that correspond to the events of another box. This lets us give events names that are meaningful to the box that contains them, on either end of a synchronization. A good example of this is shown in Figure 11 at the end of this paper, which uses renaming to map the send and receive components of events to their proper roles in the protocol. That is, a send is first mapped to a take which connects it through the interface port of Figure 11; then the take is mapped to a learn by the intruder. Figure 4 shows that the intruder's events begin with either learn or say and thus should be renamed to connect them to the send and receive of the protocol.

The boxes contained in the process regions of the interleaving boxes are interleaved, since there is no interface port symbol on the boundary between them. The events of the boxes contained in the two process regions of an interleaving box are not synchronized. For example, if the boxes $U \operatorname{ser}(b)$ and $U \operatorname{ser}(a)$ each contained an event named $a$ and both boxes performed an $a$ event, then the traces of the interleaving concurrent box containing them would include two $a$ events, not one. Even though the synchronization lines connect to the interior of both $U \operatorname{ser}(a)$ and $U \operatorname{ser}(b)$, we can tell that they do not communicate directly because the synchronization lines do not go through an interface port symbol on the region boundary between them.

Figure 3 shows that none of the boxes $U \operatorname{ser}(a), U \operatorname{ser}(b)$, or $\operatorname{Server}(j)$ communicates directly but that the three interleaved boxes are connected, via the outer parallel box, with the intruder box Intruder $(X)$. The synchronization lines connecting the boxes are abstract synchronization lines because they do not connect to specific events but are terminated inside the process region of the applicable box, without touching anything. This termination of an abstract synch line indicates that the shared event is within some greater level of detail inside the applicable box. These abstract synchronization lines are similar to the abstraction technique presented by Henderson, et al. [18] but with a different semantics. Abstract synchronization lines in a GSPML diagram, used as shown in Figure 3 have no meaning but provide a reminder of the communication pattern in the more concrete models. In a software tool these abstract synchronization lines would be place holders for the concrete synchronization lines of a more detailed view. In Figure 3 the abstract synchronization lines suggest that box Intruder $(X)$ participates in every communication event, from any of the principals.

\subsection{Intruder Structure}

Figure 4 shows the complete structure of the intruder box Intruder $(X)$. Figure 4 is an example of an external choice box indicated by a square external choice symbol on the left end of the boundary between its process regions. An external choice box offers a choice of either of its two boxes to its environment. The first event of the combination determines the choice of box.

In Figure 4 the events are named $m$ because the intruder may or may not be able to interpret the components of an event name. In Figure 4 this notation shows that the intruder box Intruder $(X)$ can copy and store details about any event communicated between the principals. This is shown by the parameterized box name Intruder (KnownFacts $\cup$ $\{m\})$. The set KnownFacts models not only the events seen by $\operatorname{Intruder}(X)$ but also any event components that Intruder $(X)$ may be able to separate and combine with components from other events. The set KnownFacts also includes any event components that $\operatorname{Intruder}(X)$ may be able to encrypt or decrypt, according to keys it already knows or learns from seeing protocol events. 


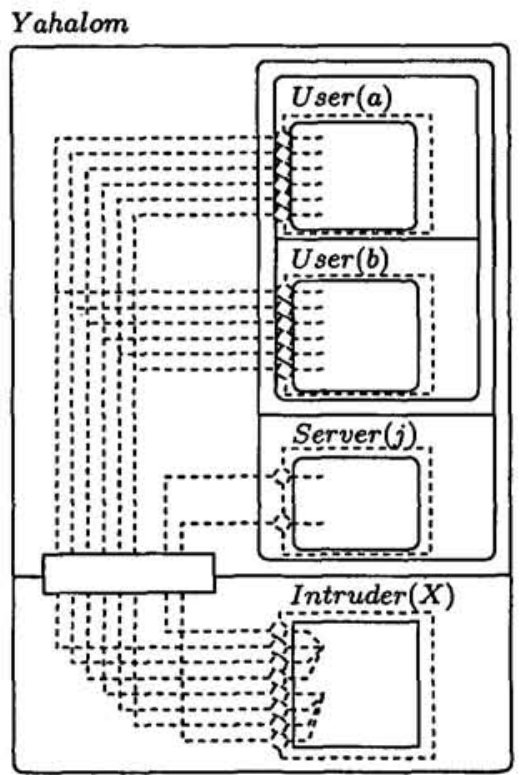

Figure 3: Yahalom: The Top-Level Structure

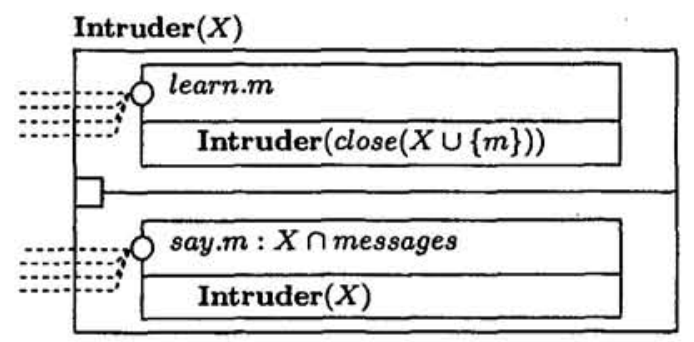

Figure 4: Yahalom: The Intruder

The use of external choice indicates that the intruder is prepared to participate in any events that any of the three other principals offers. It can either "copy" them into KnownFacts and pass them along, receive transmitted events but not relay them, or spontaneously generate bogus events that are based on the elements of KnownFacts.

Because the intruder's events are in distinct sequential processes, the intruder box does not have to make its traces of send events correspond to the traces of receive events it saw. The box following a receive event (incoming arrow) has the parameter close $(K$ nownFacts $\cup\{m\})$ that models the intruder accumulating facts in KnownFacts. The close function models the parsing, decrypting, encrypting and recomposing of events seen by the intruder. Definition of the close function is outside the scope of the GSPML language, but is represented by the parameterized box name. Use of the parameter means that there is a distinct intruder box for each possible value of the parameter.

The other part of the intruder box uses KnownFacts $\cap$ Messages to model faithful transmission as well as malicious replay and the substitution of well-formed but spurious messages by the intruder.

The meaning of the intruder's GSPML structure is that box Intruder $(X)$ must receive any event "sent" by any principal but it is not required to relay that event and may perform arbitrarily many send events before receiving an event

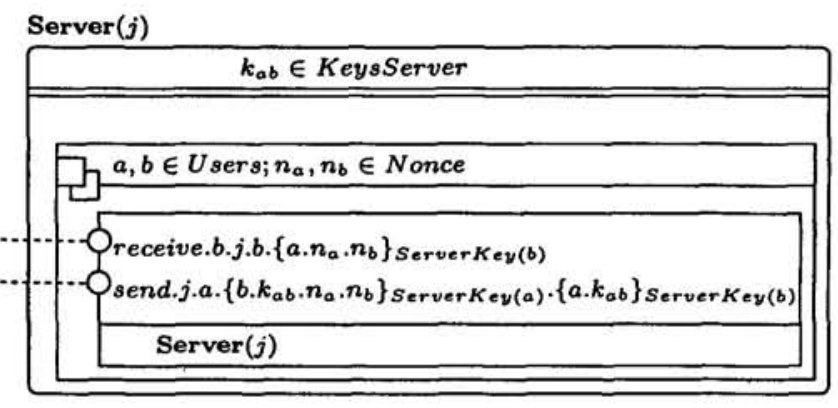

Figure 5: Yahalom: The Server Process Server(j)

from a principal.

The sequential intruder box $\operatorname{Intruder}(X)$ of Figure 4 is able to handle many events from many protocol runs because it is recursive. The box participates in one event of one protocol run, by its choice mechanism. After the single protocol event, it uses recursion to become another box that is prepared to make all of the same choices again. Recursion is defined by box names, rather than graphical notation. That is, the intruder box is recursive because its box name Intruder $(X)$ appears within the process regions of a box named Intruder $(X)$. (In our prototyping to date, we have found that purely visual modeling of general recursion is problematic.) A convention for GSPML uses a bold font for box names as a reminder that those boxes are intended to be recursive. In Figure 4 the bold font box name Intruder $(X)$ indicates the intended recursion, but GSPML attaches no meaning to the font used for the box names.

\subsection{Server Structure}

The next figure, Figure 5, shows the full definition of the server box $\operatorname{Server}(j)$. This box demonstrates several features of GSPML that we have not seen yet, including two generalized or indexed boxes. The outer concurrent box is a indexed interleaving box. It is a concurrent interleaving box because it has no interface port on the boundary between its process regions. The double line separating the two process regions tells us that it is an indexed interleaving box. The upper process region of an indexed interleaving box has a specification for the index set and the lower process region contains a parameterized box describing the processes that are interleaved. The meaning of the box $\operatorname{Server}(j)$ is that for each possible key $k_{a b} \in$ KeysServer, there is an unnamed interleaved box. The index parameter $k_{\mathrm{ab}}$ distinguishes the structural difference between each interleaved box.

In Figure 5, the each interleaved box is itself an indexed box, an indexed external choice box. An indexed external choice box allows its environment to choose from an indexed set of boxes. An indexed external choice box is depicted by the double square external choice symbol in its upper left corner. The index set is the double index $a, b \in U$ sers $; n_{a}, n_{b} \in$ Nonce. This double index shows that this indexed external choice box offers a choice of boxes over all possible pairs of users and pairs of possible nonces. The first event of each box chooses one sequential box that then performs the appropriate protocol run. This innermost sequential box of Figure 5 is essentially the same as the server box shown in Figure 2. It gives the order of the protocol steps followed by the server in a single run, with all values fixed. The box containing this single server run uses exter- 


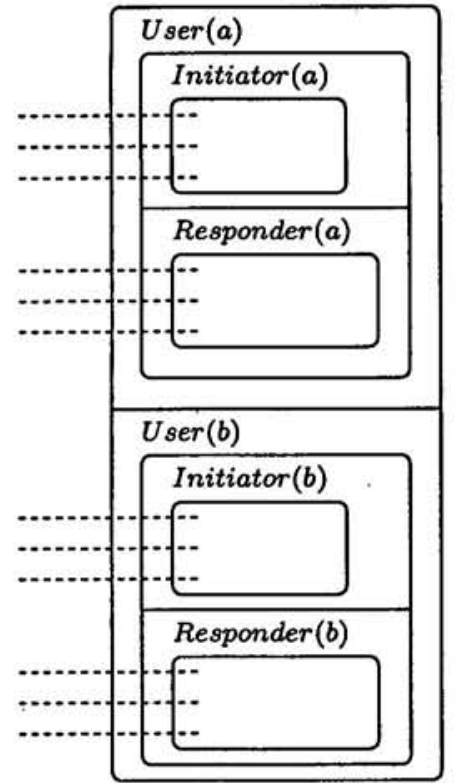

Figure 6: Yahalom: High-Level Structure of Both Users

nal choice, indexed over all possible pairs of agents and all possible pairs of nonces, to define a collection of server boxes that can conduct a single run for a fixed key $k_{a b}$, with any pair of users applying any pair of nonces. This construction models the server being prepared to engage in any run it is requested to participate in. The outer indexed interleaving box models the condition that the server $\operatorname{Server}(j)$ may be engaged simultaneously in many protocol runs, each with a different session key, including some bogus runs initiated by the intruder Intruder $(X)$.

\subsection{User Structure}

The model is completed by boxes for each of the users. In order to model a protocol of this kind, each user should be able to play either role, initiator or responder. Figure 6 shows the high-level structure of a user, either Alice or Bob. Each user is composed of two boxes, one for the user's role as a protocol run initiator and one for its role as a responder. The role modeling boxes are composed into a single user, via an interleaving box, to model the possibility of that user being engaged simultaneously in several protocol runs in either role.

Within the high-level structure of a user, the model defines the initiator and responder runs over all possible combinations of principal names, session keys, and nonces. We examine the structure of the responder role first, because it is simpler. The lower part of Figure 7 shows the box for the responder role, for user Alice. The lower part of Figure 7 does not introduce any new GSPML notation. The structure of the Responder(a) box is similar to the structure of the server box $\operatorname{Server}(j)$ shown in Figure 5: an outer interleaving box that allows a responder to be engaged simultaneously in several runs of the protocol, each distinguished by the responder's choice of nonce $n_{a}$ in the second step of the protocol. One implication of this construction is that a Responder may be engaged in several protocol runs, each run having identical values of $a, b, n_{b}$, and $k_{a b}$. While a

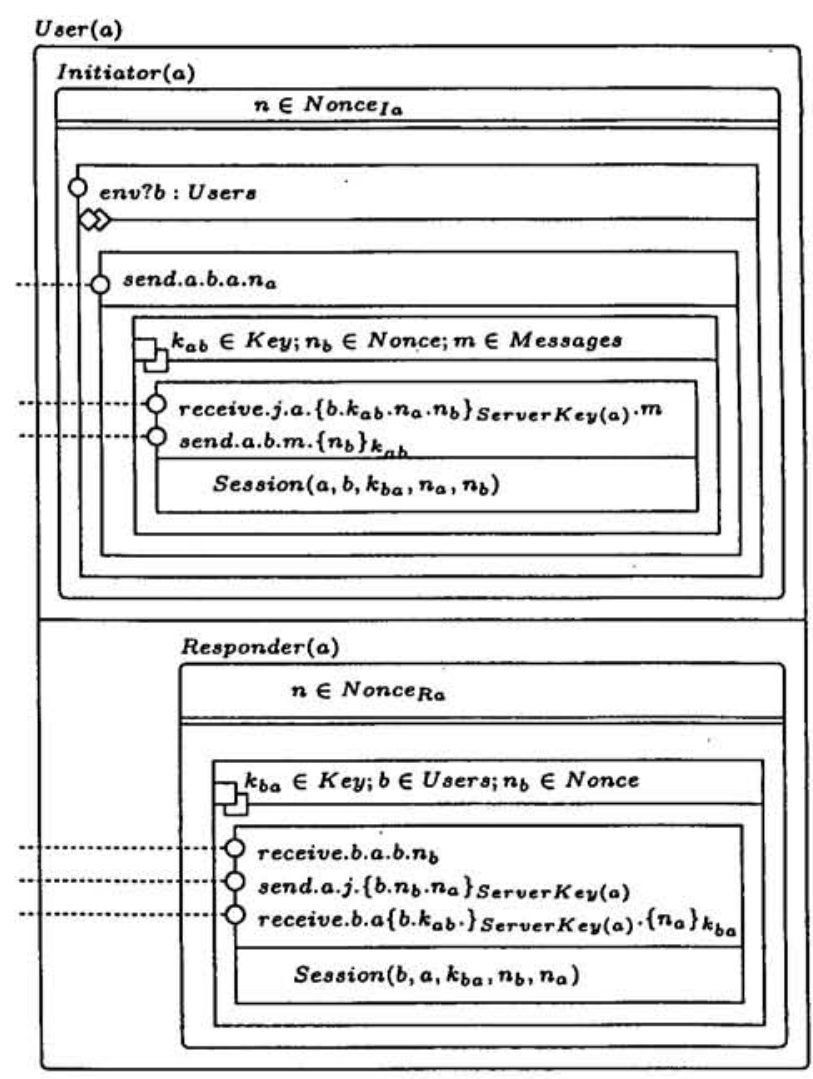

Figure 7: Yahalom: The Single User Alice

properly implemented protocol will not do this for a legitimate run, an intruder might try it. A good protocol model will be able to reflect this and support the investigation of its consequences. The indexed external choice box that defines Responder $\left(a, n_{a}\right)$ within the interleaving box gives us a choice of every possible responder process, over session keys $k_{a b}$, initiator nonces $n_{b}{ }^{1}$, and initiator names $b$. There is no recursion here; once the names, nonces, and session keys are fixed, the responder runs until a session is established, as shown by the process name $\operatorname{Session}\left(b, a, k_{b a}, n_{a}, n_{b}\right)$ in the process region of the innermost box.

The most complex component of our complete model is the initiator role. It introduces one new GSPML construct, the menu choice box. Menu choice boxes offer a choice of first events, from a single box, rather than a choice of boxes. The menu choice box of Figure 7 is contained inside indexed interleaving box Initiator $(a)$. Menu choice is denoted by the double diamond event choice symbol. Above the event choice symbol there is a single event name env?b:Users that denotes a choice of event $b$ of type $U$ sers, received from the environment env of the box. Other than this one new box, the rest of Figure 7 uses notation already explained. Notice this initial event is not connected via a synchronization line.

The added complexity in the initiator arises because of the need to model an initiator's ability to start legitimate protocol runs entirely as a consequence of its own decision. That is, the intruder Intruder $(X)$ should not be able to force any user to start a legitimate protocol run. Otherwise, the intruder either has mind control powers over the human user

${ }^{1}$ When Alice is responder, the subscripts are reversed. 
or has obtained control of the user's host. An intruder in either of these situations has no reason to try to break this session key establishment protocol. So the initiator has to use menu choice to allow its environment (i.e. the human user) to chose the responder.

The outer structure of the Initiator box in Figure 7 is similar to the responder's structure. An interleaving box models concurrent runs of the protocol using different initiator nonces $n_{a}$. Within the interleaving of runs defined by possible nonces the menu choice box models the initiator's choice of responder.

Within the process region of this menu choice box that selects a user $b$ we find a simple sequential box for each possible choice of user received from channel env. This simple sequential box transmits the applicable nonce to the chosen user's responder. The process region of this simple sequential box uses an external choice box to select the box that finishes the initiator's part of a single run, given the nonce $n_{b}$ chosen and returned by the responder $b$. Once the responder has chosen a nonce $n_{b}$ the rest of the initiator becomes a single run via a sequential box, just like the server and responder boxes seen earlier.

\subsection{Modeling a Non-Cryptographic Protocol}

We can demonstrate the versatility of GSPML by modeling a non-cryptographic security protocol, and use this second example as an opportunity to introduce further GSPML notation. In contrast to the preceding example of the Yahalom protocol, information flow security protocols do not involve cryptography. Intuitively, an information flow security protocol involves a resource that is shared between two environments High and Low. The resource is supposed to provide shared service to both High and Low but prevent information from flowing from High to Low.

The problem is not as easy as it looks and is still a research topic. One of the most difficult parts of the problem is defining absence of information flow. There are simple definitions of an information-flow-secure resource shared between High and Low that are clearly effective but inhibit or preclude functionality. For example, if the allowable security protocol provides no services to the High environment, then the shared resource in question will be information flow secure. The difficulty is getting a less restrictive definition of an allowable protocol that still has acceptable information flow properties. GSPML can both define allowable information flow and model the protocols.

We now define information flow security, for a simple service protocol. The definition of information flow security is taken from Ryan and Schneider [34]. The definition is not the best proposed by Ryan and Schneider [34] but is chosen because it can show GSPML notation we have not seen yet. Readers interested in information flow security can refer to McLean [26].

The most significant difference in this example is that we are now modeling a relation between two GSPML models. In our case, the relation is equivalence ${ }^{2}$ between the unnamed box of Figure 8(a) and the unnamed box on of Figure 8(b).

The new feature of GSPML used in this example is that both parts of Figure 8 use a hiding box. A hiding box makes

\footnotetext{
${ }^{2}$ For definitions of information flow security, the specific kind of equivalence is significant, but a discussion of that would detract from our main point.
}

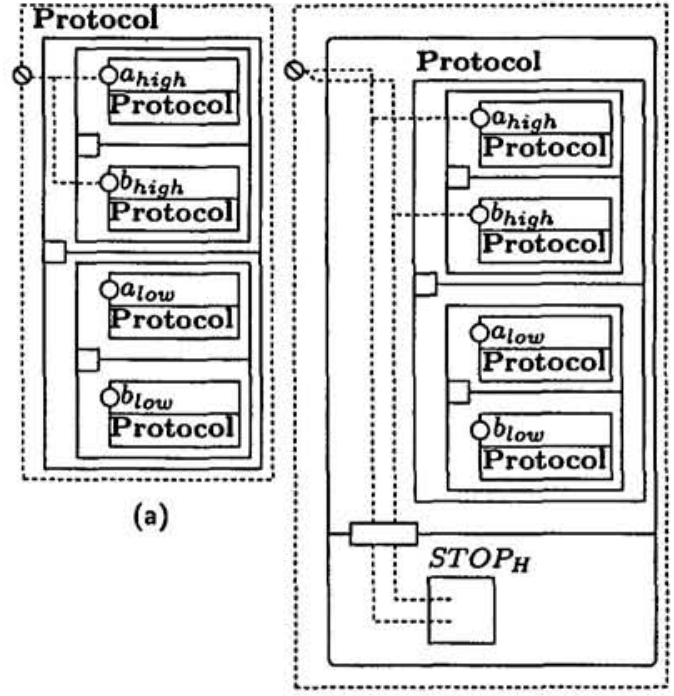

(b)

Figure 8: Modeling an Information-Flow-Security Protocol

events inside it invisible to the environment of the hiding box; inside the hiding box the hidden events are still visible. A hiding box is distinguished by its strikethrough symbols; the strikethrough symbols indicate the events that are to be hidden in the enclosed box. Outside the hiding boxes of Figure 8 events $a_{\text {low }}$ and $b_{\text {low }}$ are visible but events $a_{\text {high }}$ and $b_{\text {high }}$ are invisible. Inside the hiding box, all four events are visible when they take place.

The box construction on the right side of Figure 8 is using synchronization with the constant box $S T O P_{H}$ to block $H$ (i.e. High) events in the box Protocol. The constant box $S T O P$ is a process box that never performs events. It may be considered to have an interface with visible events, but it never performs them. Thus STOP generates only one trace: \langle\rangle$. S T O P$ does not represent normal termination but deadlock or a blocked process. When boxes are synchronized via a parallel box but the combination reaches a point in its execution where one of them is not prepared to synchronize then the combination blocks. In this case, since box $S T O P_{H}$ has precisely all the $H$ events of box Protocol, only $L$ events happen in the combination.

Figure 8 uses the process box Protocol to define the service itself and the two models containing copies of Protocol to define information flow security for the service. Essentially, the GSPML of Figure 8 defines information flow security for Protocol as the condition that any behavior of Protocol with the $H$ events hidden (i.e., Figure 8(a)) is the same as any behavior of Protocol, with its $H$ events blocked and then hidden (i.e., Figure 8(b)). The implications of this definition may be understood by considering that an arbitrary intruder box may be inserted as synchronized with the Protocol box inside each model of Figure 8; thus there is potential for different behavior to be visible between the two parts of Figure 8. For example, an adversary added to Figure 8(a) can use the external choice semantics of Protocol to selectively choose the second inner box of Protocol (the one that does $b_{\text {high }}$ ) but then only request event $a_{h i g h}$, resulting in a failure (i.e. a covert channel). A similar adversary could 
be added to Figure 8 (b) but would not be able to block the (already blocked) high events.

Adding specific details to the protocol (i.e. the Protocol box) is a key step in modeling information flow security. The example uses two simple events $a$ and $b$ while a more realistic example might use events like create-channel, start-channel, stop-channel, clear-channel and delete-channel for a multilevel boundary controller. Some specifications of Protocol will define sets of traces (and failures) that result in equality and others, sometimes surprisingly, will not. The process of designing a suitable information flow security protocol involves trade offs between the specification of Protocol, the protocol itself, and the pair of enclosing security definition boxes.

Figure 8 also demonstrates the compositional nature of GSPML models. If the service defined by the box named Protocol is to have another security property besides information flow security, then the box named Protocol can be removed unchanged from the hiding and blocking equivalence and placed in a model for that property.

\section{RELATED WORK}

After looking at two examples, it may be helpful to consider related work and analyze it according to our criteria. With our criteria: event-based, composable, comprehensive, concise, well-defined, we can assess the suitability of the various MDA/UML models for security protocol design and analysis. We can also investigate the usefulness of other modeling approaches that are not part of the MDA suite.

\subsection{UML Candidates}

To model security protocols in UML, we must use one or more of the available modeling mechanisms: actions, activities, interactions, state machines, or use cases. Use case models are high-level requirements tools and use the other visual modeling techniques to describe behavior, so they are not candidates for modeling any but the most rudimentary concepts of security protocols. UML Actions include constructs such as BroadcastSignal, ReadVariable, and WriteLink; they correspond to individual events, methods, messages, or calls. Thus, they are also not suited to modeling complete security protocols.

UML Activities organize UML Actions into structures that resemble Petri nets. UML Activities employ control- and data-flow relationships in their Petri-net-like structures, which is less desirable when the issue is protocols and we wish to avoid details about internal computations.

UML Interactions are similar to ITU Standard Z.120 Message Sequence Charts, or the older UML 1.x Sequence Diagrams: a collection of vertical life-lines with message flow between the lifelines shown horizontally. Both UML Interactions and ITU Message Sequence Charts have semantic problems. Damm and Harel have provided a well-defined semantics for these kinds of diagrams, in a visual modeling technique called Live Sequence Charts [10]. All of these "sequence-diagram" modeling paradigms have the critical strength of being event-based: they model sequences without internal computational detail. That is, they model behavior directly in terms of protocol traces. Unfortunately, they all have limited usefulness in modeling security protocols because each diagram defines only a subset of the traces of a protocol. The nature of these diagrams is that they visually enumerate traces and lack the power of set theory or process algebra to explicitly define all possible traces of a combination of principals. For example, suppose we use the BPA (Basic Process Algebra) process algebra of Bergstra and Klop [1] to define $P=a \cdot P$, the process $P$ that does event $a$ and then acts like process $P$. If the expression $\operatorname{traces}(P)$ means the set of all traces of process $P$ and the symbol denotes concatenation of traces then we can use set theory to explicitly define all of the traces of $P=a \cdot P$ as

$$
\{\langle\rangle\} \cup\{\langle a\rangle \frown t r \mid t r \in \operatorname{traces}(P)\}
$$

while the corresponding "sequence-diagram" enumeration approach is equivalent to the symbolic listing of each possible trace

$$
\langle\rangle,\langle a\rangle,\langle a, a\rangle, \ldots
$$

As soon as there is a modest variation in the pattern of the traces, this enumeration approach begins to break down. In contrast, process algebra or set theory provides us a complete definition in a single model but still allows us to unwind the model to see or check any trace. The visual modeling equivalent of set theory or process algebra is needed to solve this problem.

UML State Machines would appear to offer some promise. They are based upon (but are not the same as) the objectoriented version [17] of Harel's elegant statechart [16] visual formalism. Since statecharts are a well-defined visual model, UML State Machines should be able to define completely any security protocol, with a single model. Unfortunately, UML State Machines have some problems: 1) received events are modeled by a different mechanism that sent events, 2) the semantics are run-to-completion which poses problems for modeling some forms of recursion (Tenzer and Stevens [40] provide good examples of this), and 3) some of the events are not atomic [28]. Some of these problems are avoided by the concept of UML Protocol State $M a-$ chines. UML Protocol State Machines are like UML State Machines without UML Activities. That is, a UML Protocol State Machine only has triggers associated with its transitions while the more general UML State Machine also has UML Activities associated with its transitions. The effect of this is that a UML Protocol State Machine can describe one side of an interaction between two security principals: either the sequence of requests a principal can make or the sequence of responses that that a principal can provide. This is sufficient for constraining interfaces but not for modeling a complete security protocol.

From these circumstances we can conclude that UML is not well-suited to modeling security protocols. This leads us to examine other visual modeling techniques outside of UML, to see if they are better tools for modeling security protocols.

\subsection{Existing Visual Models Outside of UML}

We have already mentioned Live Sequence Charts as a well-defined event-based modeling technique. The problem of needing more than one diagram to define all of a protocol remains. Another possibility is a visual representation of labeled transition systems. A labeled transition system or LTS is a triple $(\Gamma, A, \rightarrow)$ where $\Gamma$ is a set of configurations, $A$ is a set of events, and $\rightarrow$ is a ternary relation: $\rightarrow \subseteq \Gamma \times A \times \Gamma$. Intuitively, the relation $\rightarrow$ represents the transitions from one configuration to another; $\left\langle\gamma, a, \gamma^{\prime}\right\rangle \in \rightarrow$ is usually written as $\gamma \stackrel{a}{\rightarrow} \gamma^{\prime}$. Labeled transition systems 
are ideal for machine representation and processing of event systems. The problem with labeled transition systems as a visual modeling paradigm is the same problem that lead to the development of statecharts: "the unmanageable, exponentially growing multitude of states, all of which have to be arranged in a 'flat' unstratified fashion" [16]. Labeled transition systems are not concise. Current LTS work is turning to algebraic treatments to overcome this difficulty. Petri nets were developed by Carl Petri [33] for formal modeling of concurrency, nondeterminism, and communication. Petri nets are well-defined and have a large body of literature. They are useful for a wide range of problems including workflow and performance modeling. The difficulty with using them to model security protocols is the presence of computation details: initial markings, places, transitions, and data flow. They are not event-based. Another difficulty is that Petrinet-based models are not naturally composable in terms of security principals.

Port state machines, a formalism developed by Mencl [28], have removed the semantic difficulties associated with UML State Machines, while retaining the semantic clarity of statecharts. Furthermore, port state machines also address modeling details needed for object-oriented programming, which the original statecharts lack. However, because of this and their state-based nature, port state machines have too much computational detail for modeling security protocols. They are not event-based.

Harel's original statecharts are a good candidate for modeling security protocols, because they lack the extra details needed to model object-oriented programming issues. They are semantically sound and can define an entire protocol with a single diagram. Statecharts also have excellent visual modeling characteristics. They are not event-based and require consideration of states and transitions as well as the events they model. We would prefer a more directly eventbased modeling paradigm.

Walters has designed RDT [42] as a formal visual language based on activity diagrams. RDT is designed foremost for visual clarity, just what is needed for visual modeling of security protocols. It would be a good candidate but it uses an LTS form of depicting behavior, so it is not event-based.

Another alternative we have not considered up to now is a graphical form of process algebra. Process algebras are event-based but avoid the explosive complexity of labeled transition systems by means of algebraic operators. Process algebras view processes as abstract trace generators and provide means for composing processes to define more complex trace generators.

Cleaveland, Du, and Smolka developed Graphical Calculus of Communicating Systems (GCCS) [8] as part of the Concurrency Factory tool [9]. The GCCS visual notation is based on Milner's CCS [31] process algebra but the diagrams are visual depictions of labeled transition systems. GCCS diagrams have the same visual limitations as basic labeled transition systems: they are not concise.

Cerone developed Visual Process Algebra or VPA [6], a modeling technique based on combinations of the CCS, CSP [21], and Circal [30] process algebras. The VPA approach models processes as boxes with ports to indicate communication and thus has the potential to be event-based. Unfortunately, VPA uses an LTS or state-machine approach within each box to model the behavior of the corresponding process. For security protocol modeling we would really

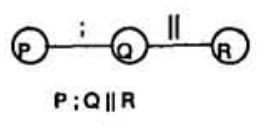

(a)
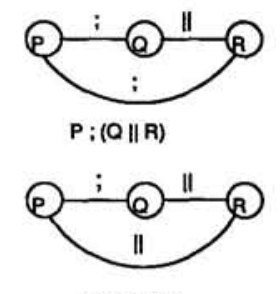

(P:Q) \|R

(b)

(c)

Figure 9: Resolving Compositional Ambiguity in gCSP

prefer an approach that avoids labeled transition systems altogether.

Gilmore and Gribaudo [13] extended the DrawNET tool to model the PEPA [20] stochastic process algebra. The DrawNET tool is oriented towards performance modeling; the graphical representation of process algebra retains the Petri nets of the underlying tool, so the DrawNET representation is not really well-suited to modeling security protocols.

The $g$ CSP (for graphical CSP) tool, developed by Hilderink, Jovanovic, et al. $[19,22]$ is the most ambitious graphical form of process algebra to date. Processes are denoted as circles in gCSP. Lines connecting the processes denote composition via the various operators of CSP. A surprising omission is the graphical modeling of events and their ordering within a sequential process. That is, even though gCSP can cleanly show sequential processes $P$ and $Q$ in parallel $P \| Q$, it cannot show the events that make up sequential process $P$ (or $Q$ ). This is not a difficulty for control applications that gCSP has been applied to, but it is critical for modeling security protocols.

From a security protocol modeling perspective, the gCSP notation is interesting because it presents a contrast to the graphical modeling paradigm proposed in this paper. Process algebras are strongly compositional. It is difficult to present complex process algebra relationships graphically. Figures 9 and 10 illustrate this difficulty in the gCSP notation. Figure 9 shows the process algebra fragment $P ; Q \| R$ which is an ambiguous term specifying the sequential (via the ; operator) and parallel (via the $\|$ operator) composition of processes $P, Q$ and $R$. Figure 9 (a) shows how this ambiguity can be drawn in gCSP. Figure 9 (b) shows how this ambiguity can be resolved by drawing cycles to add arcs for all relationships. This is problematic in complex compositions since the diagram tends to become a fully connected graph. The gCSP notation has a clever solution to this, shown in Figure 9 (c), where a smaller circle is used on one side to denote the precedence. The notation is welldefined and capable of automatic simplification. However, in complex situations, the notation becomes difficult to read, as shown by Figure 10. However, it is the lack of explicit events that renders gCSP unsuitable for security protocol modeling.

\subsection{Security Protocol Modeling Tools}

Another possibility is the (visual) modeling provided by security-protocol-specific tools. Most of the these tools have 


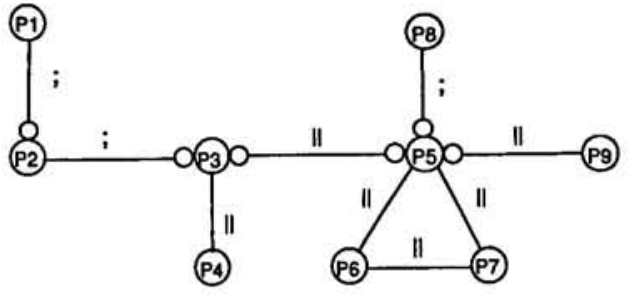

Figure 10: Complex Composition in gCSP

\subsection{UML-Based Security Modeling}

Some work has been done on security modeling with UML. Epstein and Sandhu [11] show how UML can be used to model RBAC policies. Jürjens [23] has proposed UMLsec as a means of annotating UML with sterotypes and tagged values, to specify security requirements. Basin, Doser, and Lodderstedt [2] have extended UML, via sterotypes, to Secure UML. The SecureUML language can be used to specify access control requirements on UML Class Diagrams and UML Statemachines. None of this work covers security protocol modeling. Nevertheless, it supports our observation that bare UML does not treat security issues adequately.

\section{CONCLUSIONS}

Our first conclusion is that visual modeling does not magically make complex security issues simple. The two examples were chosen because they are as complex and realistic as can be presented in a brief paper. The GSPML depiction cannot remove inherent complexity from a security protocol, but it can present security protocols in a laconic form. The fact that GSPML generates correspondingly complex models is a good thing: since simplicity and minimality are explicit design goals of security, a tool that makes adding unneeded complexity an unpleasant experience is a design aid.

Some complex concepts can be understood more quickly by visual means. Visual descriptions are sometimes preferable to text-based notation. GSPML provides those benefits for security protocols.

Our second conclusion is that GSPML is a modeling language that meets the security protocol modeling criteria: event-based, compositional, comprehensive, laconic, lucid, sound, complete, and well-defined. There is no other visual modeling technique that satisfies all of these criteria. The current Model Driven Architecture does not provide security-specific modeling facilities and its general modeling facilities fail to satisfy one or more of the security protocol modeling criteria. There are well-defined visual formalisms outside of the UML that could be used to model security protocols: labeled transition systems, Harel's original statecharts, and Petri nets. However, each of these three is also lacking according to at least one criterion.

A comment on our second conclusion is that all of the modeling approaches considered in Sections 4.1 and 4.2 are useful and in some cases superior to GSPML, for applications other than security protocol modeling. For instance, a lack of states and other internal computational details makes GSPML less suitable for modeling object-oriented implementations. GSPML is for modeling and defining protocols visually. Other than through some visual form of the rank function approach [37], GSPML is probably not suited to verification or analysis of protocols but should be used as a front-end for a protocol analysis tool as considered in Section 4.3

Our third conclusion is that, from a visual modeling point of view, the idea of a security protocol should be generalized to any form of interaction between security principals. The proposed notation should be security or protocol specific, rather than specialized to only cryptographic protocols.

Our final conclusion regards the application of GSPML. Security protocol design and modeling is usually considered a specialist responsibility, even by security specialists. 
Thus security protocols are outside the expertise of a general software developer. Why then would we need a modeling language just for security protocols? There are three reasons: 1) security specialists benefit from visual modeling, as demonstrated by the visual components of the tools described in Section 4.3 above, 2) a visual presentation may be more useful to software developers who have to implement the security protocol and thus serve as a bridge from security specialist to other developers, 3 ) many security protocols fail because they are used in new or different environments; GSPML models may reveal the impact of the new environment more clearly than a text-based model. This result is supported by the fact that (non-security-protocol) security specialists at the New Security Paradigms Workshop were able to identify protocol flaws in a few minutes, using GSPML after less than a 30 minute initial exposure to the language.

Given that fact that good well-defined visual languages are syntactic sugar for some text-based langauge we chose to use an existing semantics rather than define a new one. The essential GSPML diagramming approach is compatible with several forms of process algebra. That is, the differences in the various process algebras are not great enough to require substantially different diagrams. In our experience, we have used GSPML to visually depict security protocol models of both classical CSP and PEPA stochastic process algebra semantics. It should be possible to use GSPML-like diagrams for CCS [31] or ACP $[3,1]$ semantics.

Our future work on GSPML will include further prototyping and application, to validate the syntax, semantics, and pragmatics (e.g. laconicity). We will also strive to improve the balance [41] between security protocol complexity and the complexity of visual models drawn in GSPML.

\section{Acknowledgements}

Will Snook made substantial contributions to this work through his observations on early forms of GSPML. The comments of the anonymous NSPW referees improved the quality of this paper as did the workshop discussion. Several key ideas in this paper were brought out during discussion at the workshop and merit special acknowledgements for: Bob Blakely, Brian Snow, Simon Foley, and Steve Greenwald.

\section{REFERENCES}

[1] J. Baeten and W. Weijland. Process Algebra. Cambridge Tracts in Theoretical Computer Science. Cambridge University Press, 1990.

[2] D. Basin, J. Doser, and T. Lodderstedt. Model driven security for process-oriented systems. In Proc. Eighth ACM Symposium on Access Control Models and Technologies, Como, Italy, June 2003.

[3] J. Bergstra and J. Klop. Fixed point semantics in process algebra. Technical report, Mathematical Centre, Amsterdam, 1982.

[4] S. Brackin. A HOL extension of GNY for automatically analyzing cryptographic protocols. In Proc. 9th IEEE Computer Security Foundations Workshop, Kenmare, County Kerry, Ireland, 1996.

[5] M. Burrows, M. Abadi, and R. Needham. A logic of authentication. Proceedings of the Royal Society of London, (426):233-271, 1989.
[6] A. Cerone. From process algebra to visual language. Technical Report 01-36, Software Verification Research Centre, The University of Queensland, Queensland 4072, Australia, October 2001.

[7] I. Cervesato and C. Meadows. A fault-tree representation of NPATRL security requirements. In Workshop on Issues in Theory of Security 2003, 2003.

[8] R. Cleaveland, X. Du, and S. Smolka. GCCS: A graphical coordination language for system specification. In 4th International Conference on Coordination Models and Languages, pages 284-298, Limassol, Cyprus, 2000.

[9] R. Cleaveland, J. Gada, P. Lewis, S. Smolka, O. Sokolsky, and S. Zhang. The Concurrency Factory: practical tools for specification, simulation, verification and implementation of concurrent systems. In G. Belloch, K. Chandy, and S. Jagannathan, editors, Proc. DIMACS Workshop on Specification of Parallel Algorithms. AMS, May 1994.

[10] W. Damm and D. Harel. LSCs: Breathing life into message sequence charts. Formal Methods in System Design, 19, 2001.

[11] P. Epstein and R. Sandhu. Towards a UML based approach to role engineering. In Proc. Fourth ACM Workshop on Role-Based Access Control, Fairfax, Virginia, USA, October 1999.

[12] F. Fabrega, J. Herzog, and J. Guttman. Strand spaces: Proving security protocols correct. Journal of Computer Security, 7:191-230, 1999.

[13] S. Gilmore and M. Gribaudo. Graphical modelling of process algebras with DrawNET. In Proc. Workshop on Petri Nets and Performance Models (PNPM '03), Urbanna, Illinois, USA, September 2-5 2003.

[14] G. Guizzardi, L.Pires, and M. von Sinderen. An ontology-based approach for evaluating domain appropriateness and comprehensibility appropriateness of modeling languages. In 8th $A C M / I E E E$ Int. Conf. on Model-Driven Engineering Languages and Systems, Montego Bay, Jamaica, 2005.

[15] C. Gurr. Effective diagrammatic communication: Syntactic, semantic, and pragmatic issues. Journal of Visual Languages and Computing, 10, 1999.

[16] D. Harel. Statecharts: a visual formalism for complex systems. Science of Computer Programming, 8:231-274, 1987.

[17] D. Harel and E. Gery. Executable object modeling with statecharts. IEEE Computer, 30(7), July 1997.

[18] P. Henderson, R. Walters, and S. Crouch. Implementing hierarchical features in a graphically based formal modelling language. In Proc. 28th Int. Computer Software and Applications Conf. COMPSAC '04, pages 92-98, Hong Kong, September 2004.

[19] G. Hilderink. A graphical modeling language for specifying concurrency based on CSP. In Proc. Communicating Process Architectures 2002, Reading, England, September 2002.

[20] J. Hillston. A Compositional Approach to Performance Modelling. Cambridge University Press, 1996.

[21] C. Hoare. Communicating Sequential Processes. Prentice-Hall International, 1985. 
[22] D. Jovanovic, B. Orlic, G. Liet, and J. Broenink. gCSP: a graphical tool for designing CSP systems. In Proc. Communicating Process Architectures 2004, Headington, England, September 2004.

[23] J. Jürjens. UMLsec: extending uml for secure systems development. In Proc. UML 2002, Dresden, Germany, September 2002.

[24] P. Landin. The next 700 programming languages. $C A C M, 9(3), 1966$.

[25] R. Lichota, G. Hammonds, and S. Brackin. Verifying the correctness of cryptographic protocols using Convince. In Proc. 12th Annual Computer Security Applications Conference, San Diego, California, USA, December 1996.

[26] J. McLean. A general theory of composition for trace sets closed under selective interleaving functions. In Proc. IEEE Symposium on Research in Security and Privacy, Oakland, California, USA, May 1994.

[27] C. Meadows. The NRL protocol analyzer: an overview. The Journal of Logic Programming, 26(2):113-131, 1996.

[28] V. Mencl. Enhancing component behavior specifications with port state machines. Electronic Notes in Theoretical Computer Science, 101C:129-153, 2004. Special issue: Proceedings of the Workshop on the Compositional Verifications of UML Models, CVUML, Ed. F. de Boer and M. Bonsangue.

[29] J. Millen and G. Denker. CAPSL and MuCAPSL. Journal of Telecommunications and Information Technology, pages 16-27, March 2002.

[30] G. Milne. Formal Specification and Verification of Digital Systems. McGraw-Hill, 1994.

[31] R. Milner. Communication and Concurrency. International Series in Computer Science. Prentice-Hall, 1989.

[32] Object Management Group. Unified Modeling Language: Superstructure, Version 2.0, final adopted specification ptc/03-08-02 edition, August 2003.
[33] C. Petri. Kommunikation mit Automaten. $\mathrm{PhD}$ thesis, Bonn: Institut für Mathematik, 1962. Available as Technical Report RADC-TR-65-377, vol. 1, 1966, pages:supl. 1, English Translation.

[34] P. Ryan and S. Schneider. Process algebra and non-interference. In Proc. 12th IEEE Computer Security Foundations Workshop, Mordano, Italy, June 1999.

[35] P. Ryan and S. Schneider. Modelling and Analysis of Security Protocols. Addison-Wesley, 2001.

[36] E. Saul and A. Hutchison. Enhanced security protocol engineering through a unified multidimensional framework. IEEE Journal on Selected Areas in Communications, 21(1), January 2003.

[37] S. Schneider. Verifying the correctness of authentication protocols in CSP. IEEE Transactions on Software Engineering, 24(9):741-758, September 1998.

[38] B. Selic. The pragmatics of model-driven development. IEEE Software, pages 19-25, September/October 2003.

[39] D. Song, S. Berezin, and A. Perrig. Athena: a novel approach to efficient automatic security protocol analysis. Journal of Computer Security, 9:47-74, 2001.

[40] J. Tenzer and P. Stevens. Modelling recursive calls with UML state diagrams. In Fundamental Approaches to Software Engineering 2003, LNCS 2621, pages 135-149, Warsaw, Poland, April 2003. Springer-Verlag.

[41] E. Tufte. The Visual Display of Quantitative Information. Graphics Press, Cheshire, Connecticut, 2001.

[42] R. Walters. Automating checking of models built using a graphically based formal modelling language. Journal of Systems and Software, 71(1):55-64, 2005. 


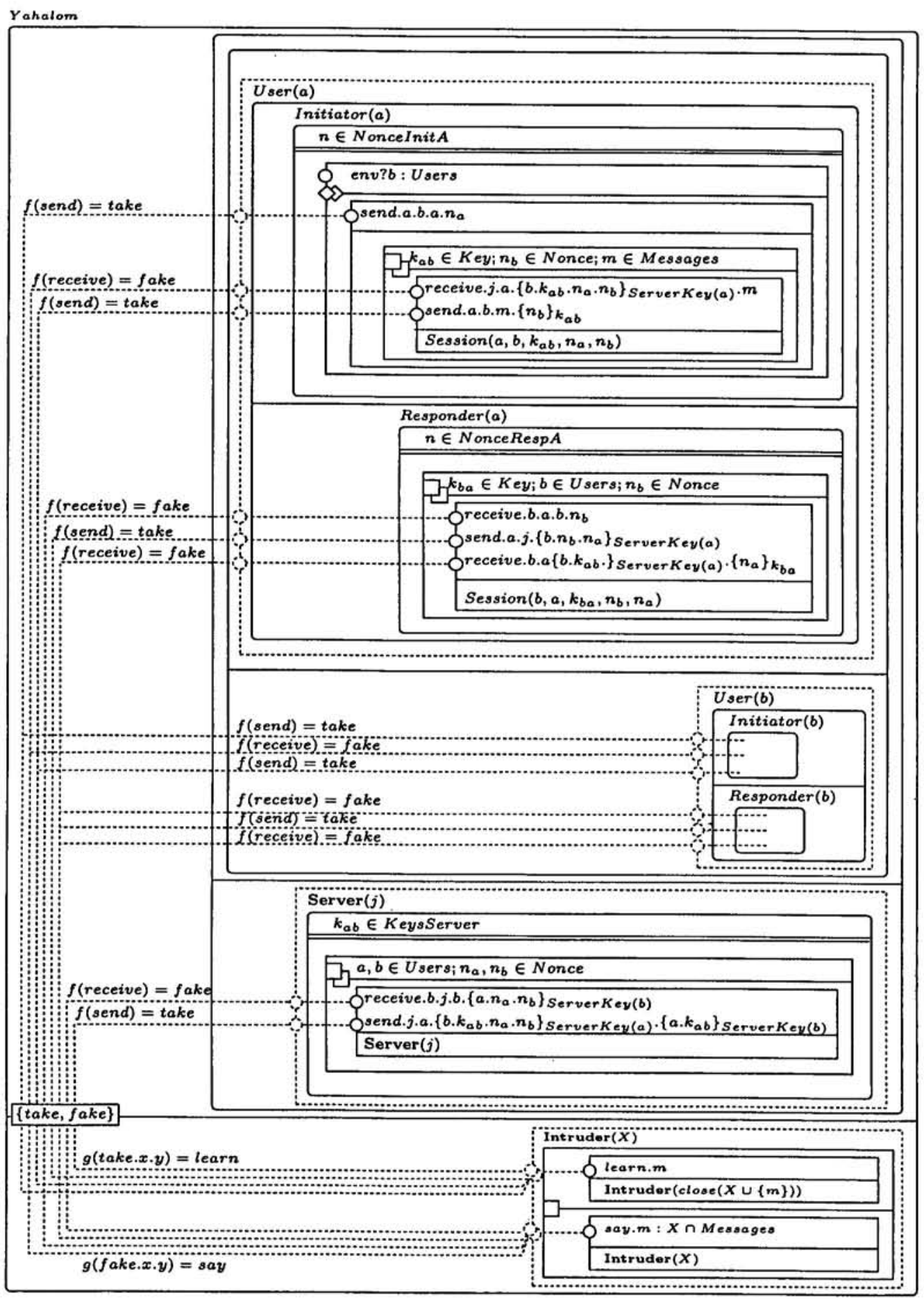

Figure 11: Yahalom: The Complete Protocol Model 